\title{
BMJ Open Oral human papillomavirus infection in England and associated risk factors: a case-control study
}

\author{
Vanessa Hearnden, ${ }^{1,2,3}$ Craig Murdoch, ${ }^{2}$ Katy D'Apice, ${ }^{2}$ Susan Duthie, ${ }^{4,5}$ \\ Nicholas J Hayward, ${ }^{5}$ Hilary Jane Powers ${ }^{1}$
}

To cite: Hearnden $V$, Murdoch C, D'Apice K, et al. Oral human papillomavirus infection in England and associated risk factors: a case-control study. BMJ Open 2018;8:e022497. doi:10.1136/ bmjopen-2018-022497

- Prepublication history and additional material for this paper are available online. To view these files, please visit the journal online (http://dx.doi. org/10.1136/bmjopen-2018022497).

Received 21 February 2018

Revised 31 May 2018

Accepted 6 July 2018

Check for updates

(c) Author(s) (or their employer(s)) 2018. Re-use permitted under CC BY-NC. No commercial re-use. See rights and permissions. Published by BMJ.

${ }^{1}$ Department of Oncology and Metabolism, The Medical School, University of Sheffield, Sheffield, UK

${ }^{2}$ School of Clinical Dentistry, University of Sheffield, Sheffield, UK

${ }^{3}$ Department of Materials Science and Engineering, University of Sheffield, Sheffield, UK

${ }^{4}$ School of Pharmacy and Life Sciences, Robert Gordon University, Aberdeen, UK

${ }^{5}$ Rowett Institute of Nutrition and Health, University of Aberdeen, Aberdeen, UK

Correspondence to

Dr Vanessa Hearnden; v.hearnden@sheffield.ac.uk

\section{ABSTRACT}

Objectives This study was conducted to determine the prevalence of and associated risk factors for infection with oral high-risk human papillomavirus (HR-HPV) in adult participants within England, and to explore any association with oral mucosal buccal epithelial cell and whole blood folate concentration.

Design This was an observational study to determine oral HR-HPV prevalence in the study population. A case-control study was performed to explore the association between infection and folate status.

Setting This study was conducted in Sheffield, UK, between April 2013 and August 2014.

Participants Seven hundred participants, aged 1860 years, were recruited from university students $(n=179)$, university and hospital staff $(n=163)$, dental hospital patients $(n=13)$, Sexual Health Sheffield patients $(n=122)$ and the general public $(n=223)$.

Interventions Participants completed a lifestyle and sexual behaviour questionnaire, provided an oral rinse and gargle sample for the detection of oral HR-HPV and an oral mucosal buccal epithelial cell sample for the measurement of oral mucosal buccal epithelial cell folate. A blood sample was collected for measurement of whole blood folate concentration.

Outcome measures The prevalence of oral HR-HPV infection in the study population was the primary outcome measure. Secondary outcome measures included associations between risk factors, folate status and infection. Results The prevalence of oral HR-HPV infection in this cohort was $2.2 \%(15 / 680)$ with $0.7 \%(5 / 680)$ positive for HPV16 or HPV18. Twenty samples were excluded due to insufficient material for HPV detection. Participants with oral HR-HPV infection were more likely to be a former smoker, and have a greater number of sexual and oral sexual partners. Folate status was not linked to likelihood of HPV infection.

Conclusions The prevalence of oral infection with HRHPV in adult men and women in Sheffield in the North of England was low. Smoking and sexual behaviour were associated with HR-HPV positivity.

Trial registration number ID14106.

\section{INTRODUCTION}

Rates of oropharyngeal cancer (OPC) are increasing worldwide, attributable to an increase in the incidence of oral infection with

\section{Strengths and limitations of this study}

This is the first report on the prevalence of oral highrisk human papillomavirus (HR-HPV) infection in men and women in England.

- The low prevalence of infection may confer only limited ability to confidently determine risk factors for oral HR-HPV infection and associations with folate status.

- Self-reported sexual, smoking and alcohol behaviours were collected using non-validated questionnaires and as such may not be accurate.

high-risk human papillomavirus (HR-HPV). ${ }^{1}$ There are large geographical variations in the proportion of OPC cases associated with HPV infection; a recent study found that $59.3 \%$ of North American, $31.1 \%$ of European and just $4.1 \%$ of South American OPC samples were positive for HPV16. ${ }^{2}$ Given the increase in HPV positive OPC, there is current interest in generating reliable data for prevalence of oral HPV infection and monitoring trends, so as to better understand aetiology of infection and progression to cancer. Greater knowledge of the prevalence of oral HPV infection is particularly timely given the ongoing debate regarding HPV vaccination in males. ${ }^{3}$

A recent meta-analysis of 29 studies worldwide, with over 22000 participants, found an overall prevalence of $2.7 \%$ oral HR-HPV infection. ${ }^{4}$ The majority of studies reporting oral HR-HPV prevalence have been performed in North or South America and there is a lack of published data available about the prevalence of oral HR-HPV in the UK. The Scottish HOPSCOTCH trial collected oral rinse and gargle samples from 380 participants and reported a prevalence of $3.7 \%$ oral HR-HPV infection in males and females aged 16-69, recruited through dental practices. ${ }^{5}$ The only study previously performed in London, England reported 5.9\% HR-HPV prevalence in HIV-negative men who have sex 
with men (MSM) ${ }^{6}$ The lack of information about oral HR-HPV prevalence for the English population constitutes an important knowledge gap.

Sexual behaviour and smoking status are considered to be the most important predictors of infection with oral HR-HPV. ${ }^{478}$ Additionally, there is evidence that the vitamin folate may influence the risk of HPV-linked cancers through mechanisms relating to HPV infection and persistence. ${ }^{9}$ Studies by our group and others have shown that low red cell folate status is associated with an increased risk of cervical HPV infection, ${ }^{10}{ }^{11}$ HPV persistence ${ }^{9}$ and cervical intraepithelial neoplasia or invasive cervical cancer. ${ }^{11}$ The association between oral HR-HPV infection and folate status has not previously been studied. Here, we have investigated the prevalence of oral HR-HPV infection in participants in Sheffield, England, and explored associated risk factors and folate status.

\section{METHODS}

\section{Study design}

A case-control study design was used to compare folate status of HR-HPV positive participants matched to three HR-HPV negative control samples; matched for gender, age and smoking status. The sample size for the case-control study was based on our data from a nested case-control study of cervical cell folate concentration in women with HR-HPV infection that persists (cases) and those in whom the virus is cleared (controls) over 6 months. ${ }^{12}$ We detected an almost two fold difference in mean folate concentration in cervical cells from cases versus controls (mean $\pm \mathrm{SD}, 5.4 \pm 4.83 \mathrm{ng}$ folate $/ \mathrm{mg}$ protein in cases vs $2.8 \pm 2.70$ controls). We calculated that 50 cases with 150 controls would allow us to detect a $45 \%$ difference between cases (oral HR-HPV positive) and controls (oral HR-HPV negative) in oral mucosal buccal epithelial cell folate, with a power of $80 \%$ and $\mathrm{p}<0.05$.

Reported prevalence values for oral HPV are extremely variable and there were few data from the UK at the time of the study. Jalal et $a l^{13}$ reported a $44 \%$ prevalence of HR-HPV in oral epithelial cells in a UK sample. Gillison et $a l^{8}$ reported an age-dependent prevalence of between $4.2 \%$ and $11.4 \%$ oral HPV infection and an average of $3.7 \%$ with HR-HPV. A sample size of 700 was selected, assuming a prevalence of $7 \%$ in the population sampled.

\section{Recruitment}

Seven hundred men and women between 18 and 60 years were recruited between April 2013 and August 2014 in Sheffield, UK. Sheffield is a city with a population of 570000 , of which approximately 50000 are students attending two universities. Sheffield has lower salaries for those in full-time employment compared with the national average; however, this is seen across many cities in the North of England. While no socioeconomic data were collected as part of this study, it is expected the results are applicable to other Northern English cities.
Participants were recruited from across Sheffield including: university students (179), university and hospital staff (163), dental hospital patients (13), Sexual Health Sheffield patients (122) and the general public (223). Recruitment was achieved through university and hospital email distribution lists, posters, patient letters, newspaper adverts and on-site recruitment at hospital locations to obtain a diverse study population.

Exclusion criteria included previous HPV vaccination, oral lesions (any lesion which would cause pain or discomfort during oral mucosal buccal epithelial cell collection), previous diagnosis of oral cancer and/or inability to understand or comply with study procedures.

\section{Sampling and questionnaire}

Participants were asked to complete a questionnaire relating to smoking status, alcohol consumption and sexual history adapted from a previous trial. ${ }^{14}$ A full copy of the questionnaire can be found in online supplementary figure 1. Example questions include:

'How many different sexual partners have you had in your lifetime?' and 'Have you ever performed oral sex on a partner?'

Each participant provided a finger-prick blood sample for the analysis of whole blood folate.

Oral mucosal buccal epithelial cell samples were collected by brushing the buccal mucosa (the cheek mucosa within the oral cavity) with an Orcellex soft brush (Rovers Medical Devices, The Netherlands) using 10 rotations of the brush on the mucosal buccal surface to collect the cells. Cells were dislodged into phosphate buffered saline and centrifuged at $250 \mathrm{xg}$ for $10 \mathrm{~min}$. Cell pellets were then weighed, resuspended in $500 \mu \mathrm{L}$ of $1.0 \%$ ascorbate and incubated for $90 \mathrm{~min}$ at room temperature. Following incubation samples were snap frozen in liquid nitrogen to aid cell lysis and stored at $-80^{\circ} \mathrm{C}$. Collected frozen oral mucosal buccal epithelial cells and blood samples were transported to the Rowett Institute of Nutrition and Health, Aberdeen, UK, for the measurement of folate using the SimulTRAC-S radioassay kit, (MP Diagnostics, supplied by Anachem, Bedford, UK).

Oral exfoliated cells from the oropharynx and oral cavity were collected for HPV detection by $30 \mathrm{~s}$ rinse and gargle with saline. HPV detection was carried out in the Cytology Department of the Royal Hallamshire Hospital, Sheffield Teaching Hospitals National Health Service (NHS) Foundation Trust, using the Linear Array HPV genotyping assay on a Roche platform, presence of $\beta$-globulin was used as a biomarker of sample validity. Samples were tested for the presence of HR-HPV subtypes 16, 18, 31, 33, 35, 39, 45, 51, $52,56,58,59,66$, defined as group 1 human carcinogens according to the International Agency for Research on Cancer along with subtype 68 , a class 2a carcinogen. ${ }^{15}$

\section{Statistical analysis}

All statistical analyses were performed using SPSS V.21. Logistic regression analysis was conducted to determine the influence of age, gender, smoking, alcohol consumption and sexual behaviour, on risk of being HR-HPV positive, using 
data from the whole dataset. Additionally, every person who tested HR-HPV positive was matched with three individuals who tested HR-HPV negative, matching for age, gender and smoking status. Logistic regression analysis was used to determine the influence of whole blood folate and buccal cell folate on likelihood of being HR-HPV positive, using data from the whole dataset and, separately, data from matched samples.
Patient and public involvement

No participants were involved with the study design.

\section{RESULTS}

The characteristics of participants recruited to the study are shown in table 1, for all participants and according to HR-HPV status.

\section{Table 1 Characteristics of study participants}

\begin{tabular}{|c|c|c|c|c|}
\hline & All $(n=700)^{*}$ & $\begin{array}{l}\text { HR-HPV positive } \\
(n=15)^{\star}\end{array}$ & $\begin{array}{l}\text { HR-HPV negative } \\
(\mathrm{n}=665)^{*}\end{array}$ & $\begin{array}{l}\text { OR for HR-HPV positivity } \\
(95 \% \mathrm{Cl})\end{array}$ \\
\hline \multicolumn{5}{|l|}{ Age (year), n (\%) } \\
\hline $18-25$ & $223(31.9)$ & $2(13.3)$ & $213(32.0)$ & 0.71 (0.06 to 7.98$)$ \\
\hline $26-30$ & 106 (15.1) & $4(26.7)$ & $101(15.1)$ & 3.10 (0.33 to 27.48$)$ \\
\hline $31-40$ & $168(24.0)$ & $5(33.3)$ & $156(23.5)$ & 2.43 (0.28 to 21.22$)$ \\
\hline $41-50$ & 123 (17.6) & $3(20.0)$ & $119(17.9)$ & $1.92(0.20$ to 18.75$)$ \\
\hline $51-60$ & $80(11.4)$ & $1(6.7)$ & $76(11.4)$ & 1.0 \\
\hline \multicolumn{5}{|l|}{ Sex, n (\%) } \\
\hline Male & $424(60.6)$ & $13(86.7)$ & 397 (59.7) & 4.39 (0.98 to 19.60$)$ \\
\hline Female & $276(39.4)$ & $2(13.3)$ & 268 (40.3) & 1.0 \\
\hline \multicolumn{5}{|l|}{ Smoking status, n (\%) } \\
\hline Never smoked & 407 (58.1) & $6(40.0)$ & $389(58.5)$ & 1.0 \\
\hline Former smoker & $180(25.7)$ & $8(53.3)$ & $166(25.0)$ & 3.12 (1.07 to 9.15$)$ \\
\hline Current smoker & $113(16.1)$ & $1(6.7)$ & $110(16.5)$ & 0.59 (0.07 to 4.95$)$ \\
\hline \multicolumn{5}{|c|}{ Units of alcohol per week, n (\%) } \\
\hline$<1$ & $153(21.9)$ & $4(26.7)$ & $145(21.8)$ & 1.0 \\
\hline $1-10$ & $241(34.4)$ & $4(26.7)$ & $233(35.0)$ & 0.62 (0.15 to 2.53$)$ \\
\hline$>10 \leq 20$ & $172(24.6)$ & $1(6.7)$ & $168(25.3)$ & $0.22(0.02$ to 1.95$)$ \\
\hline $20+$ & $134(19.1)$ & $6(40.0)$ & $119(17.9)$ & $1.83(0.50$ to 6.63$)$ \\
\hline Mean (SD) & $11.8(12.9)$ & $16.7(19.2)$ & $11.5(12.7)$ & \\
\hline \multicolumn{5}{|c|}{ No of sexual partners, n (\%) } \\
\hline $0-5$ & $332(47.4)$ & $2(13.3)$ & $330(49.6)$ & 1.0 \\
\hline $6-10$ & $173(24.7)$ & $6(40.0)$ & $167(25.1)$ & 5.93 (1.18 to 29.69$)$ \\
\hline $11+$ & $157(22.4)$ & $7(46.7)$ & $150(22.6)$ & 7.70 (1.58 to 37.51$)$ \\
\hline No answer & $38(5.4)$ & $0(0)$ & $18(2.7)$ & \\
\hline Mean (SD) & $11.4(38.3)$ & $14.4(12.2)$ & $11.5(39.3)$ & \\
\hline \multicolumn{5}{|c|}{ No of oral sexual partners, $\mathrm{n}(\%)$} \\
\hline $0-5$ & $496(70.9)$ & $5(33.3)$ & $474(71.3)$ & 1.0 \\
\hline $6-10$ & $109(15.6)$ & $8(53.3)$ & $104(15.6)$ & 7.29 (2.34 to 22.74$)$ \\
\hline $11+$ & $85(12.1)$ & $2(13.3)$ & $77(11.6)$ & 2.46 (0.47 to 12.92$)$ \\
\hline No answer & $10(1.4)$ & $0(0)$ & $10(1.5)$ & \\
\hline Mean (SD) & $6.6(13.6)$ & $8.2(7.0)$ & $6.6(13.9)$ & \\
\hline \multicolumn{5}{|c|}{ Age at sexual debut (year) } \\
\hline Mean & $17.73(3.14)$ & $17.66(3.15)$ & $17.78(3.52)$ & 1.01 (0.86 to 1.20$)$ \\
\hline Logistic regression & $\mathrm{p}=0.892$ & & & \\
\hline
\end{tabular}

${ }^{*}$ Twenty oral rinse and gargle samples contained insufficient cellular material (measured through $\beta$-globulin) to determine HPV status and were therefore omitted.

HR- HPV, high-risk human papillomavirus. OR, odds ration. SD, standard deviation. 

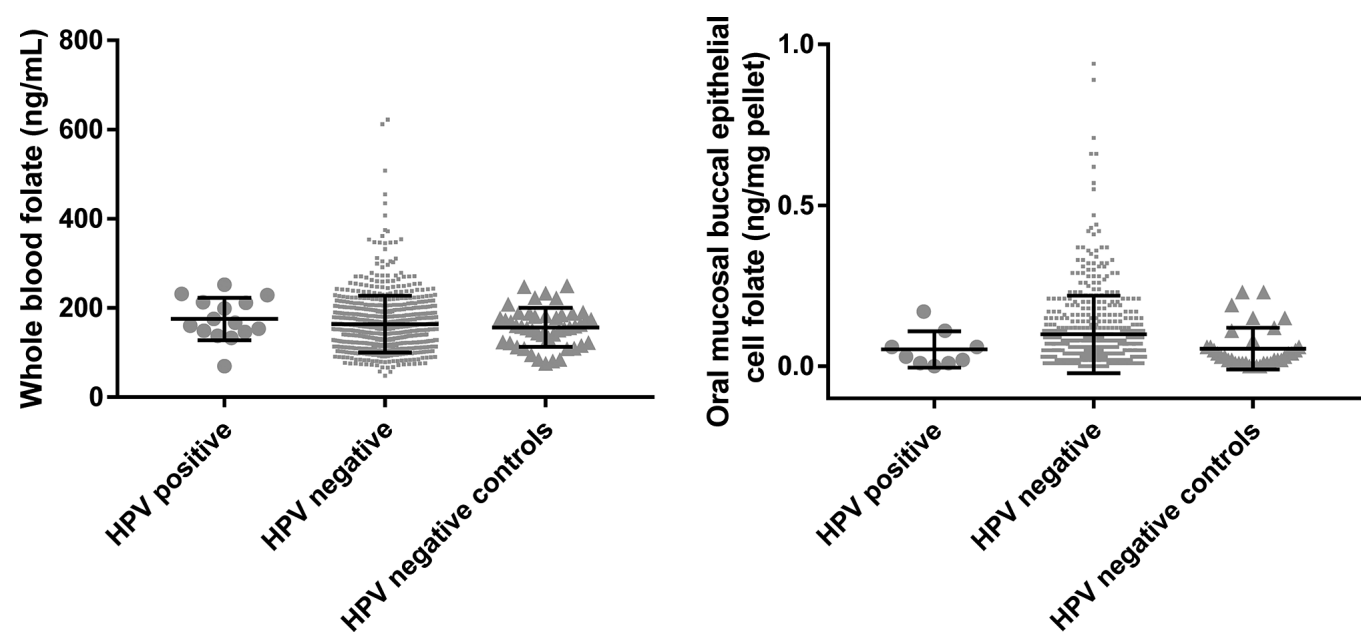

Figure 1 Measurement of whole blood folate (left) and oral mucosal buccal epithelial cell folate (right) in samples from participants positive for oral HR-HPV infection (cases), participants negative for oral HR-HPV infection and participants negative for oral HR-HPV infection (controls) matched to HR-HPV positive participants by gender, age and smoking status. No significant differences were observed. Mean \pm SD. HR-HPV, high-risk human papillomavirus.

Fifteen people $(2.2 \%)$ tested positive for an oral HR-HPV infection. Of these 4 were positive for HPV16, 1 for HPV18 and the remaining 10 participants tested positive for HR-HPV types $31,33,35,39,45,51,52,56,58$, 59, 66 and 68 (specific type not distinguishable with this detection method).

Former smokers were significantly more likely to be HR-HPV positive compared with those who had never smoked (OR 3.12, 95\% CI 1.07 to 9.15), while the sample size for current smokers was insufficient to judge risk for this population. Participants who had a greater number of sexual partners (more than six) were significantly more likely to be HR-HPV positive compared with those with fewer than six sexual partners (6-10 partners OR $5.93,95 \%$ CI 1.18 to $29.26,11+$ partners (OR $7.70,95 \%$ CI 1.58 to 37.51 ). Participants with $6-10$ oral sexual partners were also at increased risk of HR-HPV compared with those reporting fewer oral sexual partners (OR 7.29, 95\% CI 2.34 to 22.74). Participants reporting more than 11 oral sexual partners had an increased prevalence of HR-HPV (OR 2.46, 95\% CI 0.47 to 12.92), however, this did not reach statistical significance.

Whole blood folate in participants who tested HR-HPV positive was $($ mean \pm SD), $175.4 \pm 47.8 \mathrm{ng} / \mathrm{mL}$, $164.3 \pm 64.3 \mathrm{ng} / \mathrm{mL}$ in those who tested HR-HPV negative and $156.4 \pm 43.7 \mathrm{ng} / \mathrm{mL}$ in matched HR-HPV negative controls (figure 1 ).

Oral mucosal buccal epithelial cell folate concentration was $0.0524 \pm 0.0566 \mathrm{ng} / \mathrm{mg}$ cell pellet in participants who tested HR-HPV positive, $0.0514 \pm 0.0631 \mathrm{ng}$ / mg cell pellet in participants who tested HR-HPV negative and $0.0544 \pm 0.0644 \mathrm{ng} / \mathrm{mg}$ cell pellet in in matched HR-HPV negative controls (matched to HR-HPV positive by gender, age and smoking status (figure 1)). Neither whole blood folate nor oral mucosal buccal epithelial cell folate was significantly associated with risk of oral HR-HPV infection.

\section{DISCUSSION}

The prevalence of oral HR-HPV infection observed in this study (2.2\%) was in line with the global review and meta-analysis performed by Shigeishi and Sugiyama which reported $2.7 \%$ oral HR-HPV infection. ${ }^{4}$ However, the prevalence in this study was lower than two previous studies performed in the UK that reported a prevalence of $3.7 \%$ in a Scottish cohort of 380 participants attending dental clinics ${ }^{5}$ and a prevalence of $5.9 \%$ in MSM in London. ${ }^{6}$ This variation in prevalence was not surprising given the differences in study populations. Our cohort had a higher proportion of participants under the age of 25 compared with the participants in Scotland and it has previously been shown that there is a weak association between increasing age and oral HR-HPV prevalence. ${ }^{8}$ The London study population was made up of MSM and the prevalence reported (5.9\%) was comparable to previous reports of oral HR-HPV infection in MSM (6.0\%). ${ }^{4}$ These prevalence data could be considered relevant to English populations, however, given the diversity in oral HPV prevalence rates reported globally this cannot be generalised to the rest of the world.

A limitation of this study, and indeed of the many studies measuring HPV prevalence globally, is the diversity of HPV detection methods used and the HR-HPV subtypes included in detection. The assay chosen for this study was the Roche linear array HPV genotype assay that detects all 13 class 1 carcinogen HPV subtypes. The Roche linear array system was the most commonly used assay in the studies analysed in the recent meta-analysis (8/29 studies), even so the HPV subtypes detected in each of these studies varied greatly. ${ }^{4}$ Many studies have limited their analysis to HPV16 and HPV18 infection given their dominance in HPV infected OPC (and cervical neoplasia) cases, however, the data presented here showed that HPV16 and 18 were limited to just five 
participants, whereas the other HR-HPV subtypes were detected in 10 participants.

The current HPV vaccine administered to girls through the UK NHS is protective against four HPV strains (16, 18, 6 and 11). The data presented here highlight the need for future studies which investigate the relative prevalence and risk of OPC development as a result of other HR-HPV strains and the implications this may have for the future development and implementation of male vaccinations.

Previous studies have shown a strong association between oral sexual experience, number of oral sexual partners and oral HR-HPV infection. ${ }^{416}$ The results from this study confirmed this association as it showed a significantly increased risk of oral HR-HPV infection in those reporting 6-10 oral sexual partners compared with those with fewer than six oral sexual partners; those reporting more than 11 oral sexual partners showed a non-significant increase in risk; possibly due to the smaller sample size. This study also showed that total number of sexual partners was greater for people with oral HR-HPV infection, again in line with previous studies. ${ }^{416}$

Smoking is reported to be associated with an increased risk of HR-HPV infection, with a strong association in females. ${ }^{4}$ Furthermore, smoking may increase the likelihood of an oral HR-HPV infection persisting. ${ }^{17}$ Fakhry et al previously showed a strong dose-response association between tobacco exposure and oral infection with HPV16, ${ }^{18}$ however, in our study current smokers were not shown to be at significantly greater risk of HR-HPV infection. This may reflect in part the lower prevalence of smoking in this UK population (only $16 \%$ described themselves as current smokers) compared with the US population described in the Fakhry study (80\% reported using tobacco in the previous 5 days). ${ }^{18}$ This study did however demonstrate that former smokers were more likely to have oral HR-HPV infection compared with those who had never smoked.

Alcohol use was not associated with incidence of oral HR-HPV infection in our study and this is in line with other findings. ${ }^{4}$ The relationship between alcohol and HR-HPV infection appears complex with one study showing a weak association with oral HR-HPV infection and heavy drinking (before accounting for sexual behaviour $)^{8}$ and another demonstrating that those who had ever consumed alcohol were at a lower risk of oral HPV infection than never drinkers. ${ }^{19}$

Poor folate status has been linked with cancer at various sites including oral cavity and pharyngeal cancers, ${ }^{20}$ and epidemiological data are supported by plausible mechanisms. Importantly, there are also some data to suggest that poor folate status may increase the likelihood of acquiring and retaining an HPV infection. ${ }^{9}$ Furthermore, it has been shown that low folate conditions increased HPV integration into the keratinocyte host genome and reduced viral capsid production in human keratinocytes in vitro. ${ }^{21}$ In this study, we found no association between whole blood folate concentration, oral mucosal buccal epithelial cell folate concentration and oral HR-HPV infection. This may indicate that there is no causal association between folate status and oral HR-HPV infection, but the small number of participants testing positive for oral HR-HPV infection in our sample will have reduced the likelihood of detecting such an association.

There are limitations to the study, not least the difficulty of drawing conclusions about risk factors for oral HR-HPV infection when the prevalence was low and as a result CIs are broad. In addition to this, questionnaires were not validated and relied on self-reported sexual, smoking and alcohol behaviours, which may not be accurate.

To our knowledge, this is the first study to examine the prevalence of oral HR-HPV infection in adult men and women in England. The prevalence is low and the data suggest that oral infection with HR types is associated with tobacco use and sexual behaviour.

Acknowledgements The authors acknowledge the contribution by John Crossley from the Cytology Department of the Royal Hallamshire Hospital (RHH) for HPV testing and Sylvia Bates for help with recruitment from Sexual Health Sheffield at the Royal Hallamshire Hospital. The authors thank Jean Russell from Corporate Information and Computing Services, University of Sheffield, for statistical analysis and advice.

Contributors The study was conceived and designed by HJP and CM, with contribution by VH. VH took charge of the day-to-day running of the project; recruitment and collection of informed consent was the responsibility of $\mathrm{VH}$ and $\mathrm{KD} ; \mathrm{KD}$ and VH ran all clinics, collected and processed oral samples. SD and NJH developed, optimised and ran folate assays for oral buccal and RBC samples. All authors contributed to the manuscript preparation and approved the final version.

Funding This study was supported by World Cancer Research Fund (WCRF UK), as part of the World Cancer Research Fund International grant programme (Grant number 2012/587).

\section{Competing interests None declared.}

Patient consent Not required.

Ethics approval The study is approved by the NHS Humber Bridge Research Ethics Committee (REC reference 13/ $\mathrm{YH} / 0060$ )

Provenance and peer review Not commissioned; externally peer reviewed.

Data sharing statement № additional data are available.

Open access This is an open access article distributed in accordance with the Creative Commons Attribution Non Commercial (CC BY-NC 4.0) license, which permits others to distribute, remix, adapt, build upon this work non-commercially, and license their derivative works on different terms, provided the original work is properly cited, appropriate credit is given, any changes made indicated, and the use is non-commercial. See: http://creativecommons.org/licenses/by-nc/4.0/.

\section{REFERENCES}

1. Chaturvedi AK, Anderson WF, Lortet-Tieulent J, et al. Worldwide trends in incidence rates for oral cavity and oropharyngeal cancers. $J$ Clin Oncol 2013;31:4550-9.

2. Anantharaman D, Abedi-Ardekani B, Beachler DC, et al. Geographic heterogeneity in the prevalence of human papillomavirus in head and neck cancer. Int J Cancer 2017;140:1968-75.

3. Stanley M. HPV vaccination in boys and men. Hum Vaccin Immunother 2014;10:2109-11.

4. Shigeishi H, Sugiyama M. Risk factors for oral human papillomavirus infection in healthy individuals: a systematic review and metaanalysis. J Clin Med Res 2016;8:721-9.

5. Conway DI, Robertson C, Gray H, et al. Human Papilloma Virus (HPV) Oral Prevalence in Scotland (HOPSCOTCH): a feasibility study in dental settings. PLoS One 2016;11:e0165847.

6. King EM, Gilson R, Beddows S, et al. Oral human papillomavirus (HPV) infection in men who have sex with men: prevalence and lack of anogenital concordance. Sex Transm Infect 2015;91:284-6. 
7. D'Souza G, Cullen K, Bowie J, et al. Differences in oral sexual behaviors by gender, age, and race explain observed differences in prevalence of oral human papillomavirus infection. PLoS One 2014:9:e86023.

8. Gillison ML, Broutian T, Pickard RK, et al. Prevalence of oral HPV infection in the United States, 2009-2010. JAMA 2012;307:693-703.

9. Piyathilake CJ, Henao OL, Macaluso M, et al. Folate is associated with the natural history of high-risk human papillomaviruses. Cancer Res 2004;64:8788-93.

10. Piyathilake CJ, Macaluso M, Chambers MM, et al. Folate and vitamin B12 may play a critical role in lowering the HPV 16 methylationassociated risk of developing higher grades of CIN. Cancer Prev Res 2014;7:1128-37.

11. Flatley JE, McNeir K, Balasubramani L, et al. Folate status and aberrant DNA methylation are associated with HPV infection and cervical pathogenesis. Cancer Epidemiol Biomarkers Prev 2009;18:2782-9.

12. Flatley JE, Sargent A, Kitchener HC, et al. Tumour suppressor gene methylation and cervical cell folate concentration are determinants of high-risk human papillomavirus persistence: a nested case control study. BMC Cancer 2014;14:803.

13. Jalal H, Sanders CM, Prime SS, et al. Detection of human papilloma virus type 16 DNA in oral squames from normal young adults. J Oral Pathol Med 1992;21:465-70.
14. Ness AR, Waylen A, Hurley K, et al. Establishing a large prospective clinical cohort in people with head and neck cancer as a biomedical resource: head and neck 5000. BMC Cancer 2014;14:973.

15. IARC Working Group on the Evaluation of Carcinogenic Risks to Humans. Biological agents. Volume $100 \mathrm{~B}$. A review of human carcinogens. IARC Monogr Eval Carcinog Risks Hum 2012;100(Pt B): $1-441$.

16. D'Souza G, McNeel TS, Fakhry C. Understanding personal risk of oropharyngeal cancer: risk-groups for oncogenic oral HPV infection and oropharyngeal cancer. Ann Oncol 2017;28:3065-9.

17. Kero K, Rautava J, Syrjänen K, et al. Smoking increases oral HPV persistence among men: 7-year follow-up study. Eur J Clin Microbiol Infect Dis 2014;33:123-33.

18. Fakhry C, Gillison ML, D'Souza G. Tobacco use and oral HPV-16 infection. JAMA 2014;312:1465-7.

19. Pickard RK, Xiao W, Broutian TR, et al. The prevalence and incidence of oral human papillomavirus infection among young men and women, aged 18-30 years. Sex Transm Dis 2012;39:559-66.

20. Pelucchi C, Talamini R, Negri E, et al. Folate intake and risk of oral and pharyngeal cancer. Ann Oncol 2003;14:1677-81.

21. Xiao S, Tang YS, Khan RA, et al. Influence of physiologic folate deficiency on human papillomavirus type 16 (HPV16)harboring human keratinocytes in vitro and in vivo. $J$ Biol Chem 2012;287:12559-77. 